\title{
Du sperme au sang : métamorphoses de la parenté et de la société baruya (Papouasie Nouvelle-Guinée)
}

From some sperm to the blood: metamorphoses of the kinship and the Baruya society (Papua New Guinea)

\section{Anne-Sylvie Malbrancke}

\section{(2) OpenEdition}

\section{Journals}

Édition électronique

URL : http://journals.openedition.org/jso/7888

DOI : $10.4000 /$ jso.7888

ISSN : $1760-7256$

Éditeur

Société des océanistes

Édition imprimée

Date de publication : 15 décembre 2017

Pagination : 299-314

ISSN : 0300-953x

Référence électronique

Anne-Sylvie Malbrancke, « Du sperme au sang : métamorphoses de la parenté et de la société baruya (Papouasie Nouvelle-Guinée) », Journal de la Société des Océanistes [En ligne], 144-145 | 2017, mis en ligne le 15 décembre 2019, consulté le 15 mars 2021. URL : http://journals.openedition.org/jso/7888 DOl : https://doi.org/10.4000/jso.7888

\section{(c) $(\ominus \odot)$}

Journal de la société des océanistes est mis à disposition selon les termes de la Licence Creative Commons Attribution - Pas d'Utilisation Commerciale - Pas de Modification 4.0 International. 


\title{
Du sperme au sang : métamorphoses de la parenté et de la société baruya (Papouasie Nouvelle-Guinée)
}

par

\author{
Anne-Sylvie MALBRANCKE*
}

\section{RÉSUMÉ}

Partant d'une analyse longitudinale des mariages chez les Baruya de Papouasie Nouvelle-Guinée, cet article revisite leurs croyances et pratiques relatives aux substances corporelles, ainsi que les idéologies associées qui structurent les relations entre les sexes. Sans déduire de causalité mécanique entre les divers phénomènes abordés ici, je cherche à montrer que les "métamorphoses de la parenté " baruya s'accompagnent et sont reflétées par des glissements symboliques importants dans les représentations des substances vitales en jeu dans la conception de la procréation, la fabrication de la personne et le partage de l'identité. Ces recompositions sont étroitement liées à de nouvelles normes guidant les comportements sociaux et sexuels entre hommes et femmes. Je cherche à replacer cette recherche dans les débats actuels centrés sur le changement socioculturel et à envisager ces mutations de valeurs socio-culturelles en les articulant avec les permanences observées sur plusieurs plans de la vie sociale baruya.

Mots-CLÉs : Papouasie Nouvelle-Guinée, substances corporelles, relations entre les sexes, mariage, parenté, changement socio-culturel

Cet article se propose de réfléchir au rôle qu'une nouvelle perception des substances corporelles chez les Baruya de Papouasie Nouvelle-Guinée joue dans la mutation de leurs interdits matrimoniaux et dans les transformations socioculturelles relatives aux relations entre les sexes. L'articulation entre le biologique et le social est une perspective que les recherches dédiées à la Mélanésie explorèrent dès les années 1960 (Wagner, 1967 ;

\section{ABSTRACT}

This paper revisits our understanding of bodily substance beliefs and practices based on longitudinal analysis of changes in matrimonial practice and associated sociocultural transformations among the Baruya of Papua New Guinea. Eschewing mechanical causality, I attempt to demonstrate that Baruya "metamorphoses of kinship" go with and are reflected in symbolic shifts in the conceptualization of vital substances involved in the representation of procreation, the making of a person, and consubstantiality. These transformations are closely intertwined with rethought and recast norms guiding social and sexual behaviors between men and women. I attempt to inscribe this research within current anthropological debates about social change, and to approach these variations in socio-cultural values by articulating them with what appears to be strong continuity within Baruya social life.

KeYwords: Papua New Guinea, bodily substances, gender relations, marriage, kinship, sociocultural change

Buchbinder et Rappaport, 1976). Cette approche connut par la suite des développements importants, notamment dans les théories de représentation de la personne (voir entre autres Jorgensen, 1983 ; Strathern, 1988 ; Knauft, 1989 ; Bonnemère, 1990). En France, les travaux pionniers de Françoise Héritier (1994, 1996, 2003) ancrèrent la réflexion dans une conception matérialiste, partant du biologique :

* AMU, CRNS, EHESs, CREDO, Marseille. annesylvie.malbrancke@gmail.com 
" pour expliquer comment se sont mis en place aussi bien des institutions sociales que des systèmes de représentations et de pensée. » (2002 [1996] : 23)

C'est dans ce sens que Maurice Godelier analyse les articulations entre le corps et l'ordre social dans son ouvrage majeur sur les Baruya de Papouasie-Nouvelle-Guinée (1982), où il montre que l'emphase traditionnellement portée sur le sperme dans la fabrication de la personne et dans la maturation des corps servait de justification idéologique à la domination masculine, et rendait compte notamment de pratiques rituelles d'insémination de jeunes garçons ainsi que d'interdits matrimoniaux et de tabous guidant la vie sociale entre les sexes. Du moment où

"le discours sur les humeurs du corps s'inscrit toujours dans l'ensemble des représentations propre à une société » (Héritier, 1994 : 237)

il convient de réfléchir au rapport d'enchâssement de l'un par rapport aux autres, et à la relation qui s'établit entre ce premier ordre de représentation et la logique des systèmes d'alliance. Une telle réflexion trouve un terreau particulièrement fécond dans les situations de changement socioculturel, où l'analyse peut se concentrer sur une comparaison entre les différents domaines de représentations et de pratiques, pour en comprendre plus finement les ruptures ainsi que les permanences. Sans déduire de causalité mécanique entre la transformation des normes (notamment matrimoniales, mais aussi de comportements entre les sexes) et les recompositions marquant l'ensemble des représentations du biologique, je vais montrer que chez les mêmes Baruya, près de quarante ans après Godelier, c'est la justification idéologique de l'édifice de la domination masculine qui est sapée par les métamorphoses du système de parenté et de ses représentations. Si le donné biologique ne change pas (les hommes produisent toujours du sperme, les femmes ont du sang menstruel, les femmes enfantent et les hommes non), les conceptions de la procréation et de la fabrication des personnes, en revanche, connaissent des métamorphoses. Ces recompositions sont à relier à des transformations observées au niveau d'institutions sociales plus larges, autrefois légitimées et renforcées par une certaine représentation du biologique - qu'elles renforçaient à leur tour.

On cherche donc ici à penser la nature des liens de correspondance entre les représentations et les pratiques qui relèvent des rapports entre les sexes et de la parenté : s'agit-il d'une concomitance, autrement dit d'une évolution simultanée, ou bien est-il possible d'identifier un rapport de cause à effet, par lequel une transformation d'un ordre provoque un changement de l'autre ? Il faudrait, dans ce dernier cas, et s'il y a transformation structurelle logique, identifier l'enchaînement des mutations créant ce rapport, étant entendu que l'on ne peut accepter le postulat selon lequel tout se transforme toujours au même rythme : de la même façon que le vocabulaire d'une langue évoluera plus rapidement que sa grammaire, les pratiques et les représentations ne changent pas de manière simultanée. En l'occurrence, on pourrait dire que la transformation structurelle observée chez les Baruya s'apparente à un fragment de leur grammaire sociale, car les représentations des sexes codent et guident d'autres aspects de la vie sociale plus largement ; en ce sens, ce sont divers pans et plans de la vie sociale baruya qui se métamorphosent, comme je vais tâcher de le montrer.

La discussion amorcée ici s'inscrit donc dans la problématique du changement socioculturel, par le biais d'une étude longitudinale des pratiques matrimoniales et des représentations de la personne au sein d'une même société. Héritier posait déjà les jalons d'une telle réflexion : les associations établies entre divers domaines, comme la transmission du sang et les règles de mariage, créent un " cadre invariant, qui est un fait de structure"(2003 : 10). À quelles conditions ce cadre invariant change-t-il ? Car si le cadre lui-même demeure, non seulement les éléments qui le composent peuvent se modifier, mais la relation entre les différents éléments peut aussi se transformer : ceci nous renvoie à la réflexion menée par Sahlins autour des structures de conjoncture (1981). Avec l'exemple de l'arrivée du Capitaine Cook à Hawai'i, Sahlins étudie la rencontre entre l'événement et la structure afin de saisir la coexistence entre synchronie et diachronie en histoire. Si la culture établit les conditions de compréhension et de représentation du processus historique, elle se dissout et se reformule à son tour à travers la pratique (1981 : 7). Les catégories locales sont ainsi revues dans la pratique et au prisme de la conjoncture et des métamorphoses que celle-ci induit, au point parfois que la relation entre ces catégories en est altérée, et qu'un nouvel ordonnancement de la structure ainsi qu'une nouvelle compréhension de la conjoncture guideront la pratique à venir (1981 : 54).

Joel Robbins reproche cependant à ce type de modèle de ne parler de changement socioculturel qu'en termes de continuité (Robbins, 2007), et de s'engager dans la voie de la réflexion sur les permanences culturelles sans laisser de place théorique à la rupture. On ne désamorcerait cette tendance qu'en abordant le changement à partir des notions de valeurs et de hiérarchie (empruntées à Dumont) : au lieu de faire le décompte des anciens et des nouveaux constituants culturels d'une société, pour conclure à un changement ou à l'absence de changement, il faudrait obser- 
ver les relations entre les constituants (anciens et nouveaux), et leur éventuelle hiérarchie.

Cet article prend un exemple précis où s'articulent continuité dans le changement et rupture fondamentale d'avec les valeurs du passé. Je tâcherai de montrer que malgré l'existence continue de certaines logiques d'organisation sociale, des pans entiers des relations entre les sexes se sont transformés chez les Baruya, tant dans leur dimension structurelle qu'expérientielle, dans le vécu quotidien des individus. Il s'agira donc d'examiner comment les Baruya se rapportent, par les discours et dans les faits, à certaines pratiques sociales (notamment matrimoniales) et sexuelles, dont la symbolique qui les sous-tend a profondément changé, ébranlant certains principes de l'organisation sociale tandis que d'autres demeurent intacts.

Une étude longitudinale des données récoltées chez les Baruya permettra de comprendre la logique et la dynamique de ces éléments reliant les corps à l'ordre social et notamment à la pratique matrimoniale. Cette approche est rendue possible par l'analyse informatisée ${ }^{2}$ des données de mariage relevées par Godelier (archives non publiées, conservées en accès restreint sur la plateforme ODSAS - voir Dousset 2009) comparée à une analyse similaire des données dérivant de mes propres enquêtes généalogiques menées entre avril 2013 et avril 2014 dans la même vallée de Wonenara (Eastern Highlands). Mon étude comparative entre les deux moments ethnographiques a porté principalement sur les mariages dans leur détail, mais il me fut possible également de m'appuyer sur les matériaux publiés et non publiés de Godelier pour l'étude des terminologies de parenté, des témoignages relatifs aux normes de mariage, ainsi que des représentations des fluides corporels - autant d'aspects que j'ai tâché de revisiter et d'explorer dans mon propre travail. Regarder les mariages dans leur détail statistique n'implique évidemment pas de laisser de côté les normes matrimoniales propres au terrain étudié ; à l'inverse, le postulat scientifique de telles approches consiste à réévaluer les terminologies de parenté et les règles matrimoniales au prisme des comportements réels (Houseman et White, 1996). Je chercherai ainsi à mettre en regard divers ordres du discours et de la pratique, tout en tâchant d'adopter une approche diachronique de ces phénomènes, lorsque les données sont disponibles, afin d'en percevoir plus fine- ment les transformations et de les relier à celles qui marquent l'univers socioculturel baruya.

\section{"Ce n'est pas fait pour être mangé !" : les métamorphoses du sperme et du sang}

Les Baruya habitent deux vallées des Hautes Terres de Papouasie Nouvelle-Guinée et appartiennent à la famille des Anga, ensemble d'une douzaine de groupes de taille variable, dont l'histoire et la géographie communes sont attestées génétiquement et culturellement (Bonnemère, 1996 : 36-40). Ces groupes partagent nombre de caractéristiques culturelles, mais au sein desquels des différences existent (Lemonnier, 1981, 1997, 2010), notamment dans le domaine des initiations, des rapports entre les sexes, de la parenté et des rituels mortuaires. L'une de ces différences majeures, volontiers soulignée dans la littérature sur le sujet, concernait l'" homosexualité ritualisée " ${ }^{3}$, qui, inconnue chez les Anga du sud (Ankave, Bonnemère, 1996), caractérisait en revanche les initiations masculines des Anga du nord que sont les Baruya, les Sambia et les Iqwaye (voir respectivement Godelier, 1982 ; Herdt,1981; Mimica, 1981). Dans ces tribus acéphales ignorant les grands échanges cérémoniels, la vie rituelle tournait autour de la guerre et des initiations masculines, l'une comme les autres fonctionnant de manière étroitement liée, renforçant une structure sociale et psychique de solidarité masculine ancrée aussi dans la croyance en la toute-puissance du sperme (Herdt, 1981). Bruce Knauft rappelle que nombre d'ethnographies détaillées de Papouasie Nouvelle-Guinée établissent une absence de corrélation statistique entre solidarité masculine dirigée contre les femmes et guerre d'un côté, et relations homosexuelles ritualisées de l'autre (1993 et 2003 : 141). Cependant, parmi certains groupes, la concomitance de ces éléments formait système : la structure des croyances relatives aux fluides corporels allait de pair avec le fonctionnement de la structure sociale, et se trouvait renforcée et justifiée à son tour par la guerre et par d'autres expériences masculines de la vie quotidienne et rituelle (voir aussi Keesing, $1982: 34$ ).

Autrefois donc, pour les Baruya, les Sambia, les Iqwaye, et environ $10 \%$ des sociétés mélanésiennes (Knauft, 1989 : 200), le sperme était une substance majeure représentant principe de

2. Ces données furent analysées systématiquement grâce à l'emploi d'un logiciel de traitement des données de parenté, PUCK (Program for the Use and Computation of Kinship data), mis au point par Klaus Hamberger dans le cadre du groupe "Traitement informatique de la parenté » (voir Hamberger et al. [2009] pour une présentation plus détaillée de l'outil). De plus amples détails sont disponibles sur le site http://kintip.net/, où le logiciel est librement téléchargeable.

3. Gilbert Herdt fut parmi les premiers à aborder l'ethnographie des pratiques sexuelles entre individus de même sexe, sujet relativement absent de la littérature anthropologique de la Mélanésie jusqu'aux années 1980. S'il parla d'abord d' « homosexualité ritualisée ", il préféra ensuite à cette expression celle d' "insémination des jeunes garçons ", pour rendre compte de l'asymétrie entre l'âge des deux partenaires, et ne pas télescoper pratique sexuelle ritualisée et orientation sexuelle individuelle. 
vie et de force. Il pouvait notamment circuler des aînés aux cadets lors de cérémonies initiatiques où l'on pratiquait la fellation rituelle ${ }^{4}$, en grand secret des femmes (Godelier, 1982 : 9394). Il arrivait que le sperme circule du mari à sa femme pour lui redonner force et vitalité après un accouchement ou après avoir eu ses règles (Godelier, 1982: 98). Tous les individus consommaient donc du sperme à un moment de leur existence et de leur développement, car cette substance était représentée comme " la vie, la force, la nourriture qui donne la force à la vie " (Godelier, 1982 : 90).

Gilbert Herdt en particulier offre une description très détaillée des croyances collectives liées à l'ingestion du sperme, que les jeunes initiés se doivent de consommer "as regularly as eating sweet potato" (1994 [1981] : 234). Ce faisant, son analyse examine tant la construction idéologique et sociale de cet homo-érotisme rituellement construit que les expériences individuelles des jeunes novices ainsi forcés à se nourrir de la semence de leurs aînés, dans la crainte de ne pas grandir ni d'atteindre une virilité socialement et culturellement construite sans cet apport (1994: 232, 234). Chez les Sambia comme chez les Baruya, les initiations étaient pour les novices un moyen en vue d'une fin : "creating maleness and manliness» (1994: 244), ce processus qui arrive «naturellement " chez les filles, dont les règles se déclenchent à la puberté, mais qui doit être induit chez les garçons (1994: 245).

Cette identité masculine ne pouvait être atteinte, dans les constructions idéologiques des Anga du nord, qu'en excluant les femmes des rituels, pour permettre la reproduction de l'engendrement sans la mère (Godelier, 1982 : 91). Coupables d'avoir "contaminé " les jeunes garçons par la proximité avec leurs propres fluides corporels, radicalement opposés à ceux des hommes (induisant la faiblesse et la maladie au lieu de la force et de la vie), les femmes se voyaient violemment séparées de leurs fils, et ne pouvaient d'ordinaire leur adresser à nouveau la parole que de nombreuses années plus tard (1982: 63-72).

Or qu'en est-il de la circulation et de l'ingestion du sperme aujourd'hui ? Pour l'ethnographe au fait des pratiques rituelles rapportées par ses prédécesseurs, le changement est patent: le sperme, «ce n'est pas fait pour être mangé !». Je ne maventurai sur ce terrain que de longs mois après mon arrivée, quand mon intimité avec cer- tains individus était suffisamment établie pour aborder le sujet des pratiques sexuelles en causant aussi peu de gêne que possible d'un côté comme de l'autre. L'échange le plus violent à ce sujet eut lieu avec un groupe d'hommes, dont faisaient partie un vieux maître des initiations et un homme âgé d'une quarantaine d'années, chargé de résoudre les conflits et de faire appliquer la loi dans la vallée (avec qui, au demeurant, j'avais établi une relation amicale après de longues conversations et de nombreux moments passés ensemble, invariablement ponctués de blagues et de cadeaux). Or ce dernier m'intima l'ordre de me taire, de ne plus jamais aborder le sujet de ces pratiques passées. Ce qu'il montrait alors n'était pas tellement une forme d'ignorance ou de surprise à l'idée que leurs ancêtres se prêtaient à de tels actes, mais un degré élevé de honte et un refus catégorique d'associer ce passé, par ailleurs glorifié constamment par tout un chacun, avec ce que la construction contemporaine et normative de la sexualité verrait comme " dégoûtant ", déplacé - et surtout, illégal (voir aussi Knauft, 2003 : 146).

L'imagerie associée aux fluides corporels alimente des discours à la teneur très différente de ceux entendus autrefois. Car aujourd'hui, on voit dans le sperme un agent potentiellement nocif, avec lequel il faudrait limiter le contact dans les rapports sexuels. De l'admission de tous (hommes et femmes de tous âges), la fellation entre mari et femme ne se pratique plus. Dans la maison des hommes (kwalanga), les pratiques homosexuelles mentionnées plus haut se seraient arrêtées en 1960 après l'établissement des premiers Blancs dans la vallée (Godelier, 1982 : 91). Le déplacement de la symbolique du sperme est clair: de puissance génératrice de vie, il est devenu source de mort. Car dans l'ordre de l'étiologie des infections sexuellement transmissibles, la responsabilité est portée sur le sperme, qui en est devenu le principal vecteur, et dont l'absorption est par conséquent taboue. J'ai demandé à des femmes mariées de tous âges, et dont j'étais assez proche, si elles buvaient "l'eau de leur mari ", "lakala alyeu " ${ }^{6}$. Des réactions de dégoût intense $^{7}$ accueillirent ma demande, des yeux écarquillés, et quelques expressions de sympathie et d'angoisse à mon égard : bien entendu, ma question prouvait que je connaissais la pratique et il leur fallait donc m'expliquer ce à quoi je m'exposais. Quelques rires ponctuèrent parfois ma question, devant ce que mes interlocutrices

4. Notons que certaines sociétés pratiquaient la sodomie (sociétés du Mont Bosavi et de la côte sud de la Nouvelle-Guinée - voir Herdt, 1982 et Knauft, 1993), voire, moins communément, l'onction de sperme sur le corps du jeune garçon (Knauft, $1989: 218$ ).

5. L'homosexualité est punie par la loi en Papouasie Nouvelle-Guinée.

6. Les termes vernaculaires sont rapportés en italiques. Notons cependant que je ne pouvais faire de phrases complètes et mener d'entretiens qu'en tok pisin, langue véhiculaire officielle du pays avec l'anglais et le hiri motu.

7. Offrant une symétrie intéressante avec les réactions des hommes devant le sang menstruel (Godelier, 1982 : 99). 
virent comme l'ignorance de la femme blanche. Elles concluaient invariablement d'un air entendu : «ce n’est pas fait pour être mangé ! ». Je fus mise en garde contre de terribles maladies, dont les symptômes restaient à la fois vagues et hyperboliques, et dont le vecteur de contagion était clair : l'homme, le sperme.

À cette contamination par le sperme de l'homme s'ajoute l'idée que les infections sexuellement transmissibles passent également par les femmes qui couchent avec des hommes mariés (parfois pour de l'argent). Hommes et femmes stigmatisent donc également les femmes, mais seulement une catégorie d'entre elles: celles qui ne se plient pas aux exigences sociales de la "bonne conduite matrimoniale" (voir Wardlow 2006). Ces "tukina meri $\|^{8}$ sont décrites comme le "récipient " où s'accumulerait le sperme de plusieurs hommes, substance par laquelle la maladie se transmettrait ensuite de foyer en foyer, et d'autant plus délétère qu'elle échapperait aux liens du mariage. Plusieurs perspectives voient donc le sperme comme dangereux: celui que la femme ingère, même provenant de son mari, celui qui passe dans un corps de femme qui n'est pas légitimement liée à l'homme avec qui elle a des relations, et celui qui est ainsi "mélangé " à la substance d'un mari, à son insu. Corps des femmes et corps des hommes sont donc exposés aux maladies, par contraste avec la situation d'autrefois, où la force des hommes seule était menacée par le rapport sexuel avec la femme.

Très répandue notamment dans les Hautes Terres de Nouvelle-Guinée, la notion de " pollution " faisait traditionnellement de la femme un agent de contamination, dont le contact était perçu comme susceptible d'affaiblir l'homme, et de lui faire perdre des fluides vitaux (voir entre autres Hage et Harary, 1981 ; Jorgensen, 1983 ; Herdt, 1984). Les hommes baruya manifestaient autrefois envers le sang menstruel en particulier une "attitude presque hystérique, faite d'un mélange de dégoût, de répulsion et surtout de peur» (Godelier, 1982: 99). Cette substance était perçue comme sale, polluante, répugnante, et dangereuse car capable de saper cette fameuse "force " masculine.

Si aujourd'hui les hommes ne parlent pas volontiers de tels sujets, on constate que le tabou porté sur le sang menstruel perd de son intensité, sans toutefois disparaître tout à fait. Les jeunes femmes mariées me disent que les hommes n'évitent plus systématiquement les femmes quand elles ont leurs règles ${ }^{9}$, et qu'elles-mêmes ne sont pas tenues de s'isoler (sauf lorsqu'elles accouchent ${ }^{10}$ ). Les adultes des deux sexes de plus de quarante ans continuent de parler cependant des risques liés à la proximité des hommes avec une femme menstruée, et il ne leur viendrait pas à l'idée d'accepter de nourriture de leur part. En revanche, les hommes plus jeunes, surtout lorsqu'ils ont fréquenté l'école, déclarent pouvoir recevoir de la nourriture d'une femme menstruée, qui ne sera pas systématiquement vue comme un agent de contamination. Un professeur baruya de l'école primaire locale me dit un jour que selon lui il suffisait que son épouse se lave bien les mains avant de cuisiner et de lui donner de la nourriture, pour éviter de transmettre des germes. Ainsi émerge progressivement une forme de discours exogène, médical et hygiéniste, qui rapporte les effets des substances corporelles, dont on se prémunit par des gestes de purification (comme autrefois), mais qui n'inscrivent pas pour autant les personnes par leur corps dans une hiérarchisation nette. Sang menstruel et sperme doivent être évités dans des contextes particuliers et requièrent une forme de distance de la part des individus.

Aujourd'hui, loin d'être un agent contaminant par essence, le corps féminin trouve également place dans la conception locale de la croissance des foetus: les discours recueillis parmi mes contemporains montrent que la représentation de la procréation a fondamentalement changé. À l'époque des enquêtes de Godelier, il ne faisait aucun doute pour ses interlocuteurs que c'était le sperme qui fabriquait l'enfant, croyance qui s'apparentait pour l'auteur à un mouvement de "dénégation et de dénigrement des pouvoirs féminins dans le procès de reproduction de la vie» $(1982$ : 231). Or parmi mes interlocutrices de moins de quarante ans, il est de plus en plus admis qu'homme et femme ensemble fabriquent un foetus ${ }^{11}$, que c'est ensemble que les parents fabriquent les os et le sang du bébé. Cette opinion ne fait cependant pas l'unanimité: des femmes plus âgées, à l'instar de Briawinnac (probablement âgée de 50 ans), continuent de relayer l'idée que c'est l'homme qui permet de faire le sang, les os et la peau dans le ventre de la mère. Dans le même temps, Woyinna, une inter-

8. Du nom du billet de 2 kina (soit 60 centimes d'euro). On dit que la principale motivation de ces femmes adultères est de gagner de l'argent.

9. Il est utile de préciser qu'hommes et femmes baruya dorment toujours dans des zones différentes de la maison familiale.

10. La petite hutte temporaire, mundeanga, est évitée par les hommes de manière générale, sauf lorsqu’ils exercent des fonctions spécifiques, surtout médicales.

11. J'ai moins de données systématiques relatives aux opinions des hommes sur le sujet : plutôt que de parler du processus de procréation même, ils tendent à parler de la croissance des enfants, où le rôle nourricier des deux parents est reconnu à égale mesure. L'apport de la mère dans la fabrication de l'enfant est renforcé indirectement par l'idée, répétée par tout le monde, que les parents maternels sont, tout comme les parents patrilatéraux, "un même sang » avec Ego (voir Godelier, 1982 : 46 sur l'importance des parents maternels). 
locutrice de dix ans sa cadette, également non instruite et chrétienne, m'affirme que la femme seule transmet sa "force" au foetus masculin : malgré leur faiblesse proclamée, ce sont les femmes qui seraient à l'origine de cette qualité masculine, car la grossesse est le moment de cette transmission. Cette représentation indique une certaine brèche dans la construction idéologique opposant les deux sexes en deux pôles de force et de faiblesse (construction à laquelle les femmes aussi adhéraient, voir Godelier, 1982 : 124).

Par ailleurs, si autrefois on pensait que le sperme construisait et faisait croître le foetus, impliquant des rapports sexuels réguliers pendant la grossesse (Godelier, 1982 : 91) et si le lait maternel était perçu comme du sperme transformé (Godelier, 1982 : 116), mes interlocutrices me disent qu'aujourd'hui les relations sexuelles pendant la grossesse sont strictement interdites - renforçant l'idée de la dangerosité du sperme. Le lait maternel ne résulte pas d'autre chose que du corps féminin qui le produit, puisque les femmes ne font plus de fellations à leur mari. Cette substance a donc une existence sui generis et contribue à renforcer l'idée que la femme aussi fait croître l'enfant, puisqu' elle le nourrit au sein souvent plusieurs années après sa naissance.

Le sang est aujourd'hui d'autant plus volontiers inclus dans les représentations de la procréation que les connotations qui lui sont associées ont changé. À mesure que les hommes craignent moins le contact avec les femmes, dont les substances ne sont plus systématiquement considérées comme affaiblissantes, certains tabous régulant traditionnellement les relations sexuelles ${ }^{12}$ disparaissent (voir aussi Clark, 1993: 743). Les femmes m'ont volontiers parlé de l'insistance masculine, tant au sein du couple (elles se plaignent d'ailleurs d'avoir trop d'enfants et d'être battues si elles se soustraient à l'acte sexuel), qu'en dehors : le viol, phénomène inconnu autrefois, est de plus en plus répandu ${ }^{13}$, de même que les relations extra-conjugales avec des femmes qui demandent parfois de l'argent pour leurs faveurs sexuelles. Le corps féminin, de craint, est devenu désirable; personne ne trouve saugrenue (ou dangereuse) la possibilité de payer pour être en contact intime avec un corps féminin. Cet amoindrissement de la peur de la pollution féminine guide donc de nouveaux comportements sexuels et sociaux, favorisant notamment une mixité sociale plus grande et une possibilité (autant pratique que psychologique) accrue pour les hommes d'avoir accès au corps féminin (voir aussi Tuzin, 1997). Les relations sexuelles se multiplient donc, en des lieux, à des

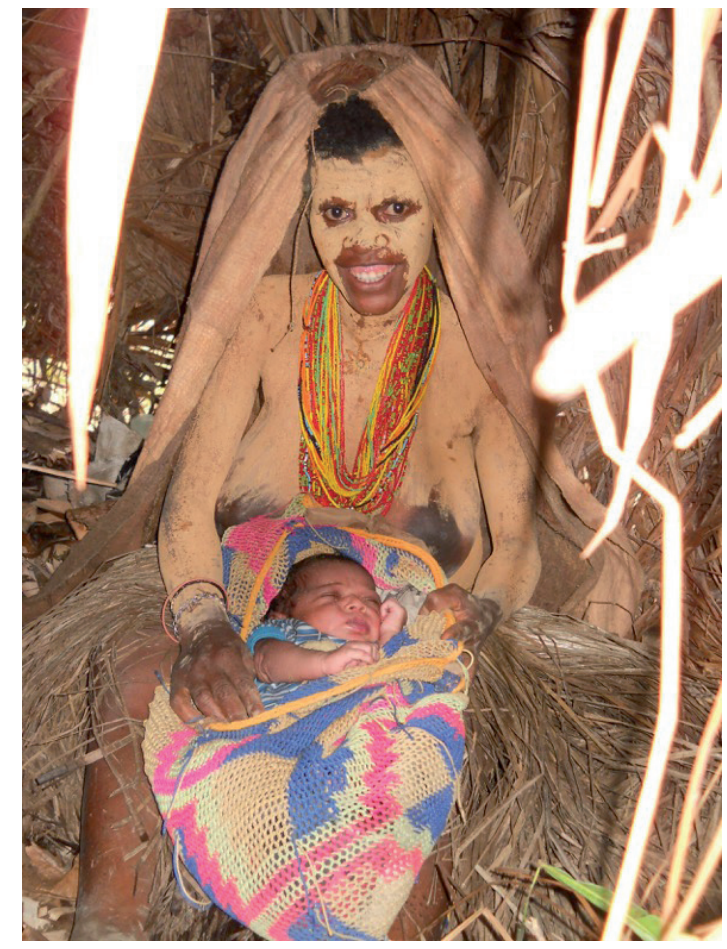

Рното 1. - Une semaine après avoir accouché de son premier enfant, cette jeune femme est prête à sortir de la mundeanga. La femme qui l'a aidée à accoucher l'enduit d'une couche de terre ocre pour, me dit-on, fortifier la jeune mère et protéger sa peau, mais aussi pour la décorer. Le bébé sera également enduit avant de quitter l'abri provisoire, village de Wuyabo, mai 2013 (C) AnneSylvie Malbrancke)

moments, et parfois avec des personnes pourtant jugés comme inappropriés - mais qui ne sont plus de l'ordre de l'« impensable».

Il est probable que cette banalisation de la proximité entre les sexes contribue à son tour à renforcer les nouvelles valeurs relatives aux corps et à leurs fluides, car une séparation franche n'est plus systématiquement indiquée (sauf à l'église, dans les maisons et à la rivière où l'on se lave), ni d'ailleurs mise en ouvre sur la base d'une peur liée à ce contact. C'est par choix que les femmes s'assoient ensemble pour discuter lors de grands rassemblements; si hommes et femmes passent plus volontiers de temps avec des individus de leur sexe, ils ne sont néanmoins pas réfractaires à l'idée de cette socialisation mixte, impensable autrefois (voir Godelier, 1982 : 29-31, décrivant les stratégies d'évitement des sexes qui n'empruntaient pas les mêmes chemins au village). La photo 2 montre ainsi deux femmes prenant part à une partie de volley-ball en compagnie de garçons. La mixité des jeux de balles n'est pas la norme, mais

12 Godelier rapporte que la dangerosité des fluides corporels féminins, ainsi que l'idée que le sperme était présent dans le corps en quantité limitée, menaient autrefois à un grand nombre d'interdits régulant les rapports sexuels au sein du couple marié (1982 : 103-104).

13. M. Nokaï, « magistrate» local, estime avoir à régler une dizaine de cas par an. 


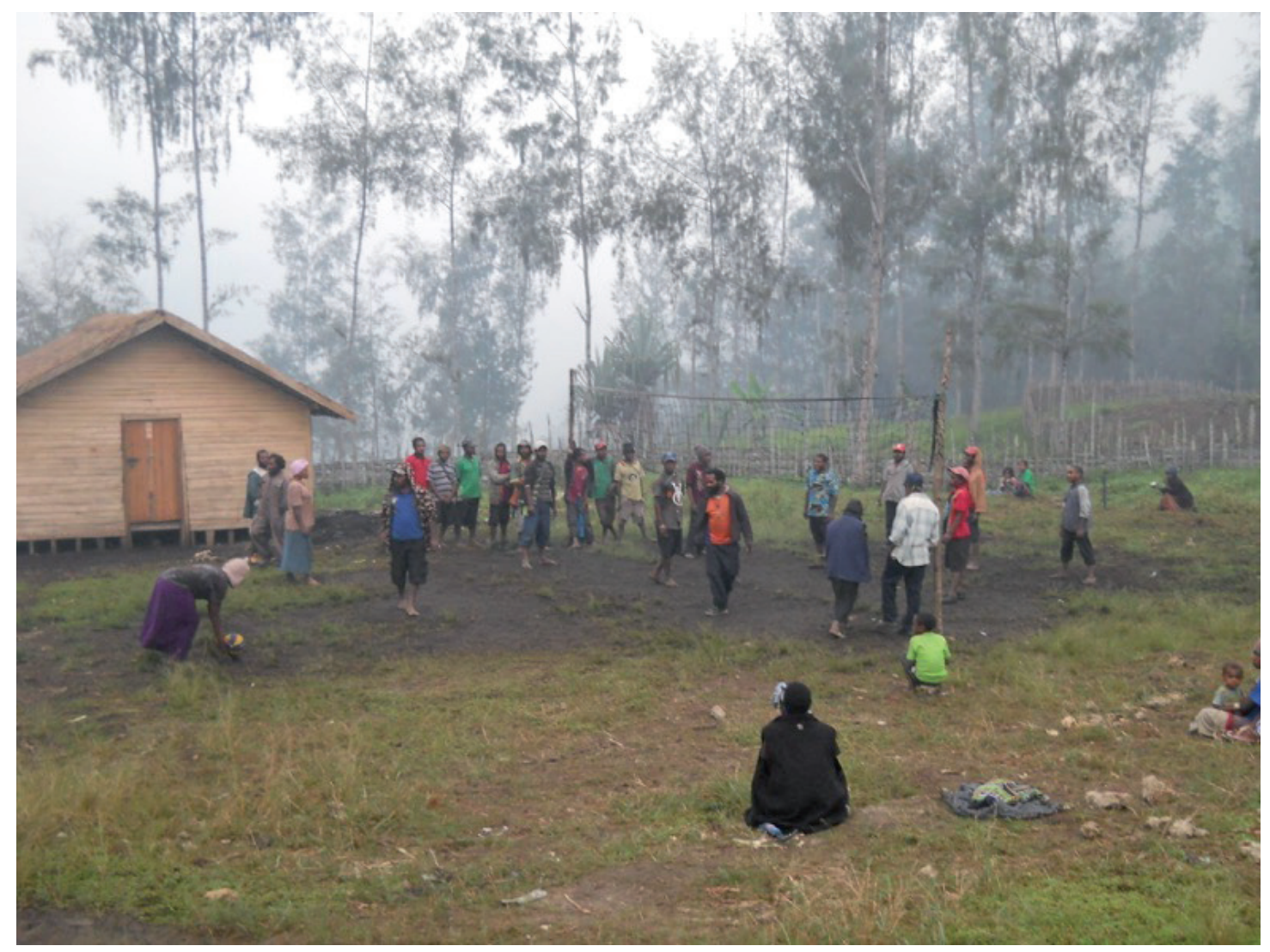

Photo 2. - Hommes et femmes peuvent participer aux jeux de balle du dimanche, parfois dans la même équipe, village de Wuyabo, mars 2014 (C Anne-Sylvie Malbrancke)

elle est une possibilité dans l'horizon du divertissement (voir aussi Lemonnier 2013 : 203-204). Certaines femmes seront trop timides pour jouer aux côtés d'hommes, et de manière générale celles qui jouent le plus volontiers ne sont pas mariées et vont à l'école (où le sport en commun fait partie du programme hebdomadaire).

De nouveaux comportements sexuels et sociaux apparaissent ainsi à mesure que des discours exogènes viennent transformer les constructions idéologiques autrefois dominantes. Ces nouvelles idées et valeurs sont transmises et renforcées par un certain nombre d'agents, parfois des Baruya et parfois des membres d'autres groupes, qui représentent des institutions arrivées dans la vallée dès les années 1960 (Godelier, 1982 : 297-335). Parmi ces individus, dont l'influence est d'autant plus importante que leur fonction est liée au prestige de la modernité (car ils ont souvent été en ville pour y être formés et certains reçoivent un salaire régulier), figurent les maîtres d'école, les pasteurs, les agents de santé ("health workers»), les représentants de la loi papoue ("magistrates»), ainsi que les élus locaux ("consols»). Ce sont presque exclusivement des hommes (à l'exception d'une femme baruya, nommée, qui représente la loi en tant que "magistrate») et même si leurs témoignages sont encore l'exception, ils sont importants pour rendre compte de l'importance grandissante de nouvelles idées et règles qu'ils enjoignent la population à respecter.

\section{Des pratiques matrimoniales en mutation}

Une précision méthodologique s'impose : comme le mentionne Pascale Bonnemère dans son étude des représentations symboliques du corps, ces thèmes ne sont pas dépourvus d'enjeux et donneront rarement lieu à des discours unitaires (1996: 221-222). Non seulement les témoignages rapportés ici ne résultent pas de discussions informelles entre les individus, mais plutôt de questions actives posées par l'anthropologue (et dont le sexe, le statut marital, l'âge peuvent orienter les réponses données), mais en plus, comme on l'a vu, les déclarations ne sont pas homogènes, selon que l'on s'adresse à un homme ou à une femme, jeune ou âgé(e), à une personne mariée ou non, instruite ou non, etc. Cependant il est intéressant de constater que dans l'ordre des discordances il n'existe pas toujours de critère déterminant pour rendre compte de l'opinion individuelle chez mes interlocuteurs 
baruya (âge, sexe, niveau d'instruction, etc.) Lorsque l'on se penche sur les discours relatifs aux mariages, on constate cette fois que les discordances sont de plusieurs ordres : non seulement elles se situent au niveau de ce que disent les individus, mais elles marquent aussi l'écart entre la norme et la pratique, ce que révèle l'utilisation du logiciel informatique, qui permet de regarder le détail de la réalité des mariages.

La population baruya s'est multipliée par un indice de 3,5 en 50 ans $^{14}$; un tel constat est pertinent pour l'analyse qui suit, car si l'on constate effectivement que les normes se transforment, on ne peut tout à fait exclure la possibilité selon laquelle la démographie serait une des clés explicatives de ces métamorphoses: dans la mesure où les partenaires potentielles sont plus nombreuses, les limites de ce que l'on considère comme des alliances "mauvaises " sont repoussées (voir cependant Porqueres i Gené, 1998). Dans le cas des Baruya, cette augmentation de la population est concomitante d'une plus grande ouverture vers l'extérieur (par le travail en plantations, les voyages en ville), permettant de sceller des alliances avec des individus d'autres groupes : j'ai pu dénombrer 74 alliances (sur les 489 recensées en 2013-2014) avec un conjoint ou une conjointe non baruya, soit $15 \%$ du total, contre les $1,1 \%$ estimés à l'époque de Godelier $(2004: 69)$.

Cela étant, j'avance ici que la recomposition des croyances relatives aux substances corporelles et à leur transmission est un facteur majeur ayant guidé et renforcé à son tour d'importantes transformations des normes et des pratiques matrimoniales baruya. Knauft rappelle qu'une telle correspondance entre mode de filiation et représentation de la conception et de la transmission des fluides corporels n'est pas systématique en Mélanésie (1989 : 205), mais cette association était observée dans la société traditionnelle baruya. Sans présumer un fonctionnement mécanique qui verrait la transformation concomitante de ces divers éléments, ce sont des aspects de la culture baruya qu'il convient de sonder à la lumière de changements socioculturels plus larges. La terminologie de parenté des Baruya, de type iroquois (Godelier, 2004 : 60), n’a pas changé, mais les conceptions des fluides corporels établis- sant le partage d'une identité de lignage se sont, elles, transformées. Traditionnellement, on ne se mariait pas dans son lignage en vertu d'une identité partagée par ses membres et transmise par le sperme du père, qui faisait donc grandir les foetus des deux sexes; transgresser ce tabou revenait à se conduire comme "des chiens" (Godelier, 1982 : 45-50), en raison d'une conjonction de substances trop semblables ${ }^{15}$ (voir Héritier, 1994 pour la théorisation du " cumul de l'identique»). Pour les Baruya, provenir de la même souche ou source impliquait le partage d'une semence issue d'une même lignée masculine. Ainsi les enfants $\mathrm{du}$ frère du père (FBCh) ne pouvaient s'épouser. En revanche, si la fille de la sour de la mère (MZD) était (est toujours) appelée "sœur", elle n’était pas interdite en mariage car elle appartenait à un autre clan (celui de son père) et n'avait pas reçu le même sperme qu'Ego. Effectivement, leurs deux pères appartenant à deux clans différents, leurs semences respectives n'étaient pas porteuses de la même identité. Il y avait là une sorte de torsion dans le système iroquois, voire une contradiction dans la structure de la parenté elle-même, en raison de la prééminence du rôle du sperme dans la définition de la personne. La FZD quant à elle, appartenant au clan qui avait reçu une femme en mariage à la génération du père d'Ego, était prescrite, notamment lorsqu'il n'y avait pas eu d'échange immédiat (Godelier, 1982 : 45-46).

Le système des substances n'était cependant pas le seul à expliquer et guider les règles de mariage : les Baruya considéraient que le mariage avec la cousine croisée matrilatérale (MBD), était "mauvais", pour des raisons stratégiques cette fois. Effectivement, par cette alliance, Ego aurait reproduit le mariage de son père et se serait privé de nouveaux alliés. On voit donc que pour rendre compte des interdits matrimoniaux traditionnels baruya, l'étude des idées sur la « fabrication" des enfants ne saurait se passer tout à fait d'une analyse des rapports sociaux et de leurs représentations locales.

Aujourd'hui la reconnaissance du rôle de la mère dans les représentations de la procréation ouvre la voie à une nouvelle conceptualisation de l'appartenance à un même lignage : celle-ci, toujours construite sur l'idée d'une transmission de substance, passe à présent par les deux sexes

14. Le Patrol report d'O. Adler de juillet 1960 indique une population de 693 personnes à Wonenara (annexe au carnet IV de Godelier) ; en 1979, le recensement effectué par Godelier (carnet II, non paginé) décomptait 952 habitants dans la vallée ; en 2013, et selon mon propre recensement, la population de Wonenara était de 2426 habitants.

15. De même, les garçons recevaient, via la fellation, du sperme de gens non apparentés en ligne patrilinéaire et en ligne matrilinéaire (Godelier, 1982 : 94).

16. On ne parle pas de sang au sens matériel, et on ne l'assimile pas uniquement aux femmes, car même si le sang a une connotation plus féminine en raison du sang menstruel, la distinction est maintenue : en langue vernaculaire, on distingue tawe, le sang, et ganié, le sang menstruel. À l'inverse d'autres exemples ethnographiques (voir Bonnemère, 1996 pour les Ankave-Anga, ou encore Busby, que dire 1997 : 264), chez les Baruya, le sang n'est pas conçu comme une substance transmise uniquement par la mère, mais précisément comme une substance passant par les deux pôles généalogiques. C'était le cas également dans le passé, mais ce n'était pas au sang que l'on prêtait la proximité identitaire entre deux individus, mais au sperme - transmis uniquement par les hommes. 
et s'exprime par l'idiome du sang ${ }^{16}$. On se dit "un même sang ", "wanpela blut ", dans tous les contextes où l'on parle de la famille élargie. Le partage de l'identité ne semble plus conditionné par la transmission d'une substance purement masculine, le sperme, mais repose sur une image associée aux deux sexes, ce qui conditionne une proximité identitaire nouvelle entre membres d'un même lignage.

Une telle modification des représentations a une incidence nette sur les interdits matrimoniaux, tant dans l'ordre du discours que dans l'application pratique de la norme. Mes interlocuteurs portent effectivement à présent l'interdit matrimonial sur toutes les femmes que l'on appelle cousines de premier degré : $75 \%$ des personnes interrogées en 2013-2014 m'ont confié ne pas aimer l'idée d'épouser quelqu'un avec qui les grands-parents étaient communs, car ils sont vus comme "trop proches", étant issus d'un "même sang". Sur le plan terminologique, les Baruya distinguent toujours les cousines croisées des cousines parallèles, mais ils ne s'appuient pas sur cette distinction pour justifier un interdit : typiquement, ils me disaient tous, en réponse à ma question sur la possibilité d'épouser une MZD,

«non, puisque c'est ma sista/sœur ! ",

et sur la possibilité d'épouser, par exemple, la FZD,

« non, puisque c'est ma kanderel cousine croisée! "

Elles sont bien distinctes, mais également interdites. Dans le même temps, les leçons de biologie dispensées à l'école remettent en cause le schéma traditionnel et amènent les jeunes gens à adopter la notion de "consanguinité " dans un sens occidental ${ }^{17}$. Ni la terminologie (termes d'adresse et de référence notamment), ni la codification des comportements à adopter à l'égard de telle ou telle classe de parentes ne changent radicalement ; de même, la filiation est toujours agnatique et les enfants appartiennent donc toujours uniquement au lignage de leur père (on est loin d'observer une quelconque transformation vers une filiation cognatique). Au sein des ensembles de représentations donc, toutes ne changent pas au même rythme.

Chez les Baruya comme ailleurs, il semble que l'on ait affaire dans ces recompositions des pratiques de mariages à une constellation d'éléments à prendre ensemble pour décrypter la situation matrimoniale. Le nouveau tabou porté sur une classe de parentes autrefois autorisées au mariage est véhiculé notamment par les représentants locaux de la loi papoue : ainsi, lors d'un tribunal de village en février 2014 constitué pour un cas de viol, le crime était d'autant plus grave qu'il réunissait deux "kandere ", deux cousins croisés (en l'occurrence un jeune homme non marié et sa cousine croisée patrilatérale, ou FZD, elle aussi non mariée). Celui que l'on appelle "magistrate» (un homme baruya), qui parlait le plus durant l'audience et apportait régulièrement des commentaires généraux sur les règles à respecter, martela, en direction du présumé coupable comme de l'assistance :

« vous êtes d'un seul et même sang, des cousins!»

Cette circonstance aggravante risquait de lui faire encourir une sanction plus lourde. Lors de l'audience, un homme s'écria à nouveau dans l'assistance :

" un seul et même sang! »,

comme pour rappeler la nature de la transgression - ce que l'on considérait à présent comme une forme d'inceste, plutôt que le viol. Car dans leur représentation de la sphère de la parenté, et en particulier dans l'ordre des proscriptions matrimoniales, les Baruya commencent à adopter cette nouvelle notion de consanguinité cognatique, et une représentation de tous les parents d'un même degré généalogique sur un même plan par rapport à Ego.

Que dire de ces préceptes dans la pratique, telle qu'elle se concrétise par les alliances conclues? Sur un corpus de taille assez importante (plus de 5000 individus), seul le recours au logiciel permet de regarder systématiquement le détail des mariages. Lors du dénombrement des mariages avec des cousines que le logiciel nomme de " premier degré " (ayant un grand-parent commun, et regroupant donc les sœurs classificatoires et les "kandere " des Baruya) il convient de relever le nombre de femmes de tel type épousées et de le comparer au nombre de femmes du même type présentes dans le corpus, donc potentiellement épousables, pour voir ce qui relève véritablement de la préférence, et ce qui n'est qu'un effet de la démographie (voir Hamberger et al., 2004).

Les indices de préférence permettent de constater une certaine cohérence entre norme matrimoniale et pratique, dans la mesure où les cousines proportionnellement les moins épousées $\mathrm{du}$ corpus (FBD et MBD) sont celles sur lesquelles portaient autrefois les interdits les plus forts. Ces mêmes indices augmentent clairement pour la MZD et la FZD, traditionnellement permises, voire prescrites, en mariage. 
La lecture du deuxième tableau (mêmes calculs opérés sur le corpus agrandi) doit se faire de manière comparative : la proportion de mariages ajoutés au total (représentant une augmentation de $25 \%)$ ne permet pas de modifier les indices comme celui de la préférence. Ici c'est bien la progression en termes numériques qui nous intéresse : que les mariages avec la MBD aient conservé un même indice de préférence n'est pas parlant, mais constater que leur nombre n'a pas du tout augmenté l'est nettement plus.

On constate ainsi que les deux cousines parallèles (FBD et MZD) sont aujourd'hui complètement évitées: pas une seule alliance avec ces femmes n'a été recensée.

\begin{tabular}{|c|c|c|c|c|}
\hline Standard & Individus & \% Indiv & Mariages & Préférence \\
\hline FBD & 44 & 22,28 & 22 & 60,94 \\
\hline FZD & 74 & 38,59 & 37 & 164,92 \\
\hline MBD & 38 & 20,11 & 19 & 77,14 \\
\hline MZD & 46 & 25,00 & 23 & 128,97 \\
\hline
\end{tabular}

Tableau 1. - Recensement des mariages avec une cousine de premier degré dans le corpus Godelier (pour un total de 1945 mariages)

\begin{tabular}{|c|c|c|c|c|}
\hline Standard & Individus & \% Indiv & Mariages & Préférence \\
\hline FBD & 44 & 20,5 & 22 & 61,98 \\
\hline FZD & 88 & 42,5 & 44 & 167,13 \\
\hline MBD & 42 & 20,5 & 21 & 77,36 \\
\hline MZD & 46 & 23,00 & 23 & 109,34 \\
\hline
\end{tabular}

Tableau 2. - Recensement des mariages avec une cousine de premier degré dans le corpus élargi (pour un total de 2434 mariages)

C'est là un changement majeur : autrefois, la MZD était la deuxième cousine la plus épousée, après la cousine croisée patrilatérale (FZD); on peut postuler qu'en raison de cette nouvelle représentation de la transmission de l'identité (par le sang plutôt que par le sperme), les préférences matrimoniales se sont modifiées.

Les cousines classificatoires, quant à elles, ont toujours eu un statut différent dans l'horizon matrimonial baruya: Godelier rappelle que le clan n'est pas une unité exogame, et que

" les mariages avec une cousine parallèle patrilatérale très distante ne sont pas interdits. » (1982: 46)

En ce sens, la logique de transmission des substances guidant la compréhension locale de l'inceste ne s'appliquerait pas traditionnellement aux cousines classificatoires (de type FFBSD par exemple). Mon hypothèse sur la consubstantialité ne s'étend pas aux cousines classificatoires parce qu'elles ont déjà été "repoussées " d'un cran hors de la sphère des sœurs, ce qui était le cas aussi dans la tradition baruya ${ }^{18}$.

La reconnaissance des liens utérins se traduit donc dans les représentations de la procréation et dans la pratique matrimoniale. En ce sens, la conclusion à laquelle arrive Bonnemère est particulièrement adaptée ici :

" on peut supposer que lorsque les théories du corps - et partant de la procréation - attribuent un rôle important aux femmes, non seulement l'image de la féminité est plus positive, mais encore les liens de parenté par les femmes sont reconnus à part entière. " (1996:391-392)

Et l'on peut émettre l'hypothèse selon laquelle leur statut dans la société se transforme à son tour (voir Bonnemère, 1996 : 381 sur cette corrélation chez les Anga), car les valeurs se sont modifiées.

\section{La domination masculine en question}

Si l'on suit Godelier, il convient de s'interroger sur la légitimité d'une hiérarchisation plaçant les hommes au-dessus des femmes dès lors que celle-ci est attaquée en son fondement, sa justification en nature : la supériorité du sperme, qui

"explique et légitime en même temps la domination masculine. » (1982: 99)

À mesure qu'une telle construction idéologique se modifie, on peut supposer que la structure sociale se métamorphose également: la modernité baruya se caractérise ainsi par une transformation du contexte politique, économique et matériel, mais surtout par un changement idéologique permettant aux femmes de trouver une place plus grande quautrefois pour être vues et entendues. Malgré certains problèmes liés à la quantification du changement dans les relations entre les sexes (voir Clark et Hughes, 1995 : 317), il n'est pas impossible de comparer la situation des femmes baruya d'autrefois à celle d'aujourd'hui, surtout si l'on ne se contente pas d'un décompte des changements ou des perma-

18. Notons que les hommes interrogés sur la question distinguent la "sista tru tru ", " sœur véritable ", soit la germaine ou la cousine parallèle), de la femme qui est " comme une sœur mais un peu différente " ("wankain olsem sista, tasol em $i$ narapela liklik») et que l'on peut par conséquent épouser. 
nences mais que l'on cherche à évaluer les transformations des valeurs et leur hiérarchisation au sein de la société étudiée (Robbins, 2007).

Se pencher sur le rôle et la place des femmes implique de regarder dans le détail la façon dont l'échange des sœurs - reformulé à l'aune de valeurs "modernes" de choix individuel et d'amour - et les initiations masculines - amputées de certains rituels traditionnels - créent une structure sociale particulière au sein de laquelle la capacité d'action des femmes, ainsi que leur représentation idéologique, sont radicalement repensées. Une hypothèse formulée par Godelier reliait ainsi l'échange direct de femmes aux initiations masculines ${ }^{19}$, qui auraient créé

" une sorte de force collective qui se trouve derrière chacun d'eux, toujours présente et menaçante lorsqu'ils disposent du destin personnel de leurs sœurs ou de leurs filles. » (1992: 20)

Notons d'emblée la moindre fréquence, voire la disparition, des rituels d'initiation féminine baruya (sanginié) qui, traditionnellement, visaient

« à imprimer dans l'esprit des jeunes filles la nécessité et la légitimité de la subordination des femmes. " (Godelier, 1982: 89)

Pour Bonnemère, l'absenrobinsce d'initiations féminines est à relier à l'absence de pratiques homosexuelles ritualisées chez les Ankave pour rendre compte de la moindre domination masculine (1996:381-382). Plus largement, et partant des ethnographies menées dans la région, Bonnemère contraste deux types d'initiations masculines observées au sein de l'ensemble culturel anga : celles où la domination masculine était reproduite, et celles qui maintenaient l'asymétrie entre les sexes sans la légitimer (1996 : 379). La situation baruya contemporaine s'éloigne du premier cas de figure pour se rapprocher du second. Le premier stade des initiations masculines, la muka, eut lieu en mon absence en décembre 2013. Chez les Baruya, il semble que ces rituels se recomposent sans tout à fait disparaître (contraster avec Tuzin, 1997) : encore aujourd'hui, nul ne saurait se soustraire aux initiations, dont la compatibilité avec la fréquentation d'une Église est constamment réaffirmée (voir Lemonnier, 2013 et Malbrancke, 2016). Selon mes interlocuteurs (des hommes mariés), certains rituels traditionnels n'ont plus lieu systématiquement - comme percer la cloison nasale des jeunes garçons - et s'ils affirment que la maison des hommes est toujours le lieu de la "kalta», de la "culture » locale et de ses enseignements moraux, ils se plaignent cependant volontiers que cette "kalta" n'est plus la même qu'autrefois, qu'elle n'est plus "entière" (" $i$ no full blo mipela»). Malgré le maintien d'une certaine "solidarité masculine", notamment par l'aspect secret de ces rituels et l'infliction de sévices corporels aux jeunes initiés (voir Godelier $1982: 124)^{20}$, les initiations n'instaurent plus un fossé infranchissable et prolongé entre les sexes. Après quelques mois de réclusion, les jeunes gens reprennent vite le cours normal d'une vie sociale mixte à l'école, au village, aux soirées " disco » (où la promiscuité entre jeunes de sexe opposé est le but avoué des réjouissances).

Les épreuves physiques et psychologiques semblent peu de choses aux hommes les plus âgés, qui répètent à l'envi, et selon une rhétorique assez banale, qu'ils sont les derniers dépositaires d'une culture en voie de disparition, que les rituels contemporains ne sont "rien", que les garçons ne sont plus initiés "comme il faut " et n'ont pas l'ardeur de leurs ancêtres à la guerre ou aux jardins et ignorent les mythes ancestraux. Il leur semble aussi que les jeunes gens se marient "n'importe comment", selon leur bon plaisir : effectivement, si l'échange de sœurs, le ginamare, existe toujours dans l'univers matrimonial des Baruya, son contenu et sa signification pour les intéressés ont été profondément reformulés. Moins qu'un arrangement en amont par les familles, où les jeunes gens se plieraient à la logique du don réciproque entre deux lignages, il sagit à présent de négociations ad hoc, qui ne fonctionnent et ne sont mises en application que si les intéressés donnent leur accord - et en premier lieu les femmes, le plus souvent à l'origine d'un mariage ou de son annulation. La pratique récente mais répandue du "brideprice", ou compensation matrimoniale, contribue également à l'augmentation des mariages qui ne sont pas arrangés dès l'enfance mais découlent plutôt d'un choix. Les femmes baruya se disent plus libres de quitter leur mari, car elles ne craignent plus de mettre en péril par ricochet l'alliance de leur frère. Elles voient aussi dans le brideprice une valorisation de leur statut d'épouse et insistent pour que leur mari verse rapidement cette somme d'argent à leur lignage. En ce sens, la situation baruya contraste nettement avec celle documentée dans d'autres régions du pays, où l'adoption du "bridewealth " ou "brideprice" mène à une augmentation des violences conjugales liées à l'idée d'un "achat " de la femme, sur laquelle un mari penserait auto-

19. Voir Bonnemère (1996 : 390-391) pour une analyse de cette idée, à partir du cas ankave.

20. Les hommes avaient d'abord minimisé l'ampleur des sévices infligés aux jeunes garçons dans la maison des hommes, que je ne découvris que parce que deux d'entre eux me furent amenés pour recevoir des soins suite à des blessures similaires sur la jambe ; questionnés, les hommes m’avouèrent qu'ils frappaient violemment les jeunes initiés en pleine nuit pour leur apprendre à se réveiller en sursaut et à faire face à une incursion ennemie. 
matiquement avoir des "droits» (voir notamment Eves, 2006; Wardlow, 2006). La sphère matrimoniale n'est plus un espace de domination pour les femmes, dont les trajectoires individuelles ne dépendent plus du choix des hommes (Godelier, 1982 : 221).

Cette autonomie individuelle est renforcée par la place qu'elles trouvent aujourd'hui dans diverses sphères de la vie sociale, au premier chef l'école, ancien privilège masculin (Godelier, 1982 : 304 ; voir aussi Bonnemère, 2016 et Lemonnier, 2013 pour un historique détaillé de la chronologie relative à l'école dans la vallée).

La lecture du tableau 3 montre que le nombre de filles présentes à l'école diminue considérablement à mesure que l'on monte de niveau ("grade»), au point de passer d'une dominante féminine dans les petites classes à un rapport de une fille pour cinq garçons dans les classes les plus avancées. Cette table démontre l'impact de l'entrée en vigueur récente de la "free education policy ${ }^{21}$ qui rend l'école primaire gratuite pour tous et permet aux parents de ne pas avoir à pénaliser leurs filles, plus utiles dans les jardins et à la maison. Cela reflète aussi une certaine fragilité du statut des petites filles à l'école, car il suffirait que cette politique de gratuité du primaire soit remise en cause pour que leur accès à l'éducation soit à nouveau compromis.

La présence féminine à l'école n'est pas sans lien avec le rôle conféré aux femmes dans la vie politique de la communauté - à la fois au sens de leur rôle dans le fonctionnement de la communauté et dans une acception plus restreinte de pratique $\mathrm{du}$ pouvoir. Bonnemère montre ainsi que la fermeture de l'école locale de 1970 à 2007 eut pour résultat à long terme de limiter la possibilité de prise de parole par des femmes qui ne maîtrisaient pas bien le tok pisin et qui n'avaient pas la confiance suffisante pour assumer de rôle public (2016 : 214). Selon tous les individus interrogés sur le sujet, la Constitution ${ }^{22}$ affirme le droit des femmes à "bosim ol", à donner des ordres à la communauté. Les femmes peuvent, en principe, se présenter tous les cinq ans à l'élection du gouvernement local («consol»); en pratique, cependant, aucune femme n'a jamais été élue, et l'on attendrait de toute candidate qu'elle fût instruite, alors que plus de la moitié des candidats masculins aux élections de 2013 étaient analpha-

bètes. Le rôle public accordé aux femmes, reflété dans leur rôle et leur influence dans les négociations et dans le processus de prise de décision à différentes échelles (village, vallée, tribu), n’est pas établi par des discours normatifs uniformes, et la prise de parole publique féminine dépend du choix d'individus mais n'est pas culturellement attendue.

Si la propriété de la terre reste uniquement masculine, les femmes peuvent vendre le produit de leur propre travail (notamment la récolte de café), et il n'est pas rare qu'elles gèrent l'économie $\mathrm{du}$ foyer. Les femmes baruya en tant que groupe se voient allouées des sommes d'argent (variables selon les années et les fonds disponibles au niveau du gouvernement local) pour réaliser un ensemble de travaux pour la communauté (nettoyer telle portion du territoire, creuser de nouvelles latrines, etc.). De telles initiatives n'ont pas systématiquement mené à une nouvelle conception des rôles dévolus aux deux sexes, et sont parfois à l'origine d'une caricature des transformations en cours, car les hommes se plaignent de se voir " soumis » aux décisions des femmes.

La division du travail (les femmes s'occupent des tâches répétitives quotidiennes, les hommes de ce qui relève de tâches collectives plus ponctuelles) ne s'accompagne pas de discours formulés en termes de supériorité - chacun a des responsabilités différentes, et chacun doit s'en acquitter, sous peine de voir son conjoint ou sa conjointe entrer dans une colère noire et l'exprimer à coups de poings ou de bâton. La coopération entre mari et femme tend à s'instaurer en droit pour les tâches ménagères, et certaines activités ne sont plus l'apanage d'un seul sexe :

21. Ou "Tuition Fee Free policy" (TFF), introduite en 2012 en Papouasie Nouvelle-Guinée, et le premier ministre O’Neill s'est engagé à ne pas revenir sur cette décision pendant toute la durée de son mandat. Il a été réélu aux élections générales de 2017.

22. "Mama lo " en tok pisin, expression que la majorité de mes interlocuteurs avaient adoptée mais en l'interprétant, à tort, comme signifiant que cette loi (au contenu vague) conférait des droits spéciaux aux femmes ("mama " est un terme courant pour parler d'une épouse) - ce qui irritait beaucoup certains hommes. 


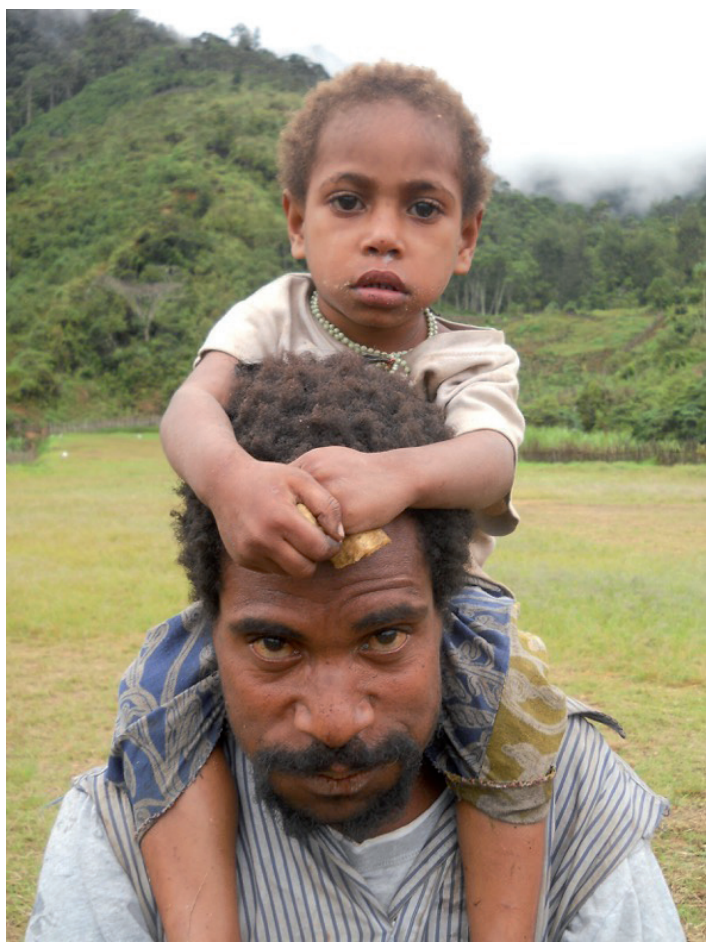

Рното 3. - Timothy et sa fille de quatre ans, Djabila, village de Wuyabo, avril 2014 (C) Anne-Sylvie Malbrancke)

on voit notamment des hommes fabriquer des filets de portage (bilums) et s'occuper d'enfants en bas âge (voir par contraste Godelier, 1982 : 105). Les femmes ont légitimement accès aux mêmes plaisirs et loisirs que les hommes (jeux de cartes et de balles notamment), auxquels elles prennent part parfois en compagnie d'hommes, notamment pour ce qui est des jeunes générations; les hommes raillent d'ailleurs ce qu'ils perçoivent comme le désœuvrement de leurs sœurs ou épouses (qui se promèneraient "sans but, comme les hommes ", "raun raun nating, olsem man "). Les différentes sphères de la société ne sont plus systématiquement assignées à un sexe ou à l'autre ; la variabilité de certains comportements au sein d'une même famille et à l'échelle de la vallée montre aussi que cette division du travail ne procède pas d'une position idéologique fixe, transmise lors d'initiations où des discours normatifs enseignent à chaque sexe à se comporter vis-à-vis de l'autre, mais dépend de plus en plus de choix individuels.

La barrière qui autrefois marquait les relations entre les sexes semble de plus en plus poreuse, et les facteurs qui contribuent à cette transformation se situent sur un continuum; les pratiques sont à la fois sous-tendues et renforcées par des représentations qui peuvent certes créer des ruptures mais participent aussi de logiques sociales propres à une société à un moment donné. Ainsi Bonnemère démontre que, chez les Baruya, l'arrivée du christianisme n'est pas en soi un fac- teur suffisant pour rendre compte d'une rupture totale d'avec les pratiques du passé en termes de relations entre les sexes : effectivement, là où officie un pasteur baruya, ses idées et orientations culturelles guideront sa réticence à laisser une place aux femmes pendant les rituels (2016: 219). À l'inverse, un pasteur extérieur à la tribu sera plus à même de leur donner une plus grande visibilité à l'office ; ce nouveau rôle et cette prise de parole publique sont importants pour renforcer les idées récentes sur l'égalité entre hommes et femmes (discours par ailleurs porté à l'unanimité par les cinq pasteurs interrogés en 2013-2014 - mais mis en œuvre de manière variable, comme le montre Bonnemère [2013 : 237]). C'est ce continuum même de transformations (partant des relations décontractées entre garçons et filles à l'école, aux jeux collectifs, aux transactions économiques mêlant les sexes) qui mène sur la voie d'une égalisation progressive entre les sexes - ou plutôt devrait-on parler d'une réduction de l'antagonisme qui marquait autrefois leurs rapports. Mais, comme le dit Joel Robbins, de telles reconfigurations doivent être pensées plutôt comme des conséquences involontaires de changements dans les valeurs que comme un modèle d'égalité entre les genres complètement articulé et énoncé comme tel (2009 : 127).

\section{Conclusion}

Loin d'être réfractaire à une pensée du changement social, Françoise Héritier posait que les "cadres invariants" formés par le système de parenté et la représentation de la personne pouvaient se manifester sous des formes diverses selon les sociétés, et se modifier dans le temps; cependant, de telles métamorphoses étaient contraintes par des logiques structurelles, toute modification n'étant pas possible ou pensable (2002 : 55-56).

L'exemple baruya confirme ces postulats car les changements observés chez les Baruya ont une cohérence interne certaine, qui s'accorde avec la covariation d'élément observée plus largement chez les Anga (voir Bonnemère, 1996: 378). Que la diminution de l'importance du rôle masculin dans la procréation et dans la croissance des personnes soit concomitante avec une amélioration (relative) de la situation des femmes comme ensemble social renforce l'hypothèse d'une corrélation étroite entre règles d'alliance, représentation de la procréation, et rapports entre les sexes.

Il faudrait encore se demander à quelles conditions et dans quelles circonstances certaines idéologies se modifient : en termes d'axiologie, ou de théorie des valeurs, on constate chez les Baruya une grande transformation relative à la 


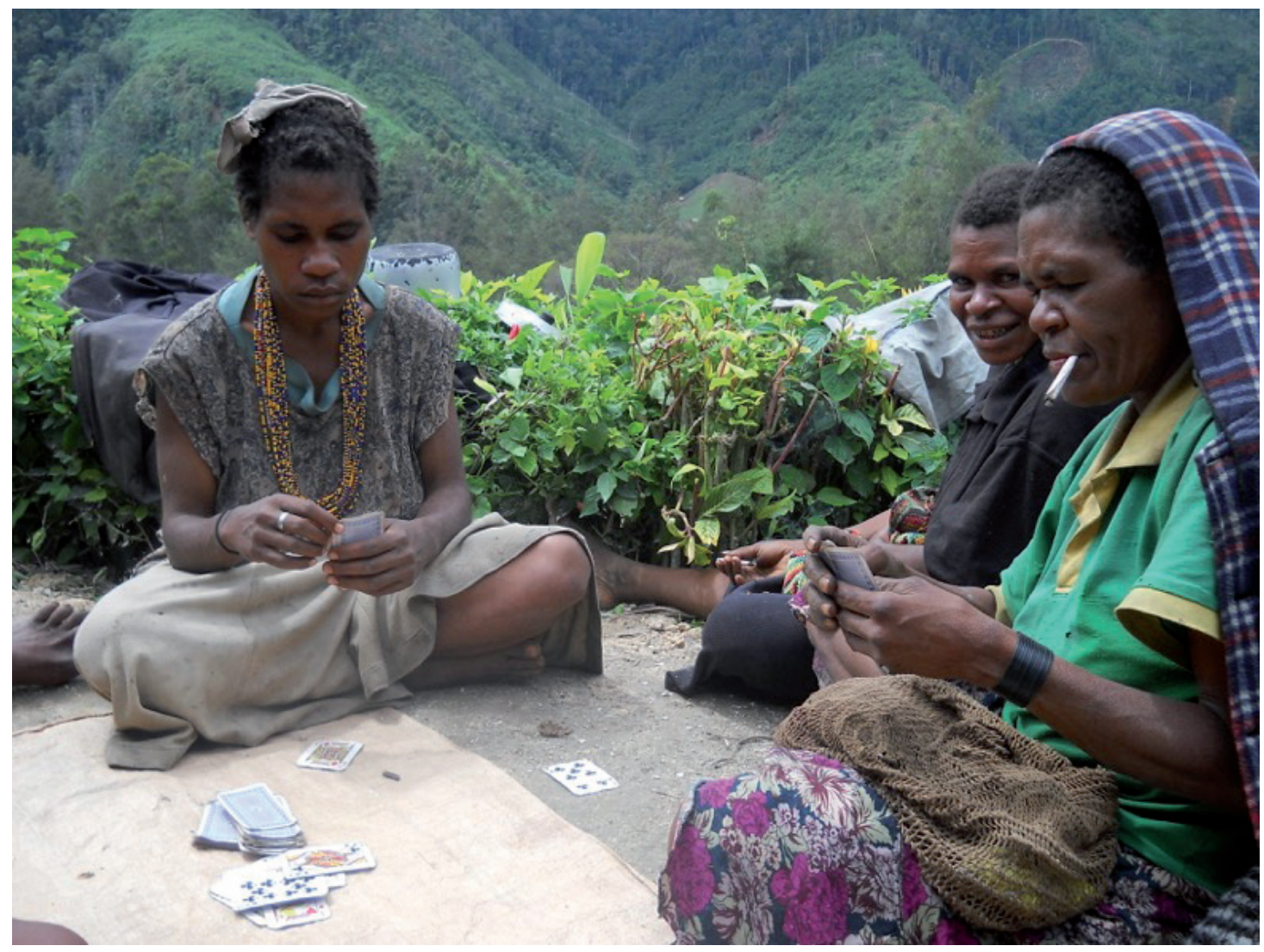

Рното 4. - Un moment de détente entre femmes, village de Wuyabo, août 2013 (@ Anne-Sylvie Malbrancke)

valence de la substance masculine et, partant, une révision de son rôle dans la conception de la procréation, dans ce qui " produit » des hommes et des femmes. Mais si la transmission du "sang " passe par les hommes et par les femmes, la transmission sociale d'éléments cruciaux pour la reproduction symbolique et matérielle du groupe (identité lignagère, terres horticoles et terrains de chasse) est quant à elle toujours uniquement agnatique. On voit donc que filiation et représentation des substances ne sont pas liées de manière systématique : le décalage entre les deux ordres de réalité est d'autant plus visible aujourd'hui que l'on peut analyser les permanences telles qu'elles s'articulent aux changements. Une approche longitudinale combinée à une analyse de la hiérarchie des valeurs propres à une société semble permettre d'avancer sur la voie de la compréhension du changement socioculturel.

Chez les Baruya comme ailleurs, les pratiques modifient les représentations car l'expérience transforme les valeurs de la société, mais l'on peut supposer que cette nouvelle expérience (par exemple la mixité des sexes) n'est rendue possible que par une métamorphose des représentations des corps masculins et féminins (qui minimise, par exemple, l'idée selon laquelle la proximité avec les femmes est un danger pour l'homme). Cependant, pour penser le changement socio- culturel sur la longue durée, il semble moins pertinent de postuler la prééminence d'un élément sur les autres (les transformations socio-économiques à la racine d'un changement idéologique), ou de chercher l'élément déterminant en dernière instance (l'événement qui transforme la structure), que de chercher à s'approcher d'une compréhension processuelle de ces phénomènes. Car ce sont les expériences de la vie sociale et des relations entre les sexes qui créent des changements dans les valeurs, dont la répétition et la sédimentation viennent à leur tour transformer la structure.

\section{Remerciements}

Mon terrain de thèse se déroula d'avril 2013 à avril 2014 dans la vallée de Wonenara (Eastern Highlands, Papouasie Nouvelle-Guinée), sous la direction de Pascale Bonnemère, que je remercie pour sa relecture fine d'une version antérieure de cet article et pour ses nombreux conseils et commentaires constructifs. Toute maladresse ou erreur demeure bien sûr de mon fait. Je souhaite remercier tous mes interlocuteurs et interlocutrices baruya, ainsi que Maurice Godelier, qui eut la grande générosité de me laisser explorer 
ses archives. Mon terrain ne fut possible que grâce à l'aide et à l'organisation en amont de Pierre Lemonnier, dont les conseils ont été très précieux à chaque étape ; qu'il en soit vivement remercié.

\section{BIBLIOGRAPHIE}

Bonnemère Pascale, 1990. Considérations relatives aux représentations des substances corporelles en Nouvelle-Guinée, L'Homme 114, pp. 101-120.

—, 1996. Le Pandanus Rouge. Corps, différence des sexes et parenté chez les Ankave-Anga, Paris, CNRS-Maison des sciences de l'homme.

—, 2001. Two forms of masculine ritualized rebirth. The Melanesian body and the Amazonian cosmos, in Gregor Thomas A. and Donald Tuzin (eds), Gender in Amazonia and Melanesia: an Exploration of the Comparative Method, Berkeley, The University of California Press, pp. 17-44.

—, 2013. À chacun sa Bible. Styles de prêche et rapport à Jésus dans la vallée de Wonenara (Papouasie Nouvelle-Guinée) ", in Christophe Pons (éd.), Jésus, moi et les autres. La construction collective d'une relation personnelle à Jésus dans les Églises évangéliques: Europe, Océanie, Maghreb, Paris, CNRs Éditions, pp. 227-258.

—, 2016. Church presence and gender relations in the Wonenara valley (Eastern Highlands Province, PNG), The Australian Journal of Anthropology 27 (2): Gender and Christianity in Melanesia: Towards a Unified analysis (M. MacCarthy and A. Eriksen eds), pp. 206225.

Buchbinder Georgeda et Roy RAPpaport, 1976. Fertility and Death among the Maring, in Paula Brown and Georgeda Buchbinder (eds), Man and Woman in the New Guinea Highlands, Washington D.C., American Anthropological Association, pp. 13-25.

Busby Cecilia, 1997. Permeable and Partible Persons: A Comparative Analysis of Gender and Body in South India and Melanesia, The Journal of the Royal Anthropological Institute 3 (2), pp. 261-278.

Clark Jeffrey, 1993. Gold, Sex, and Pollution: Male Illness and Myth at Mt. Kare, Papua New Guinea, American Ethnologist 20 (4), pp. 742-757.

Clark Jeffrey et Jenny Hughes, 1995. A History of Sexuality and Gender in Tari, in Aletta
Biersack (ed.), Papuan Borderlands: Huli, Duna, and Ipili Perspectives on the Papua New Guinea Highlands, Ann Arbor, The University of Michigan Press, pp. 315-340.

Dousset Laurent, 2009. odsas: Online digital sources and annotation system for the social sciences (http://www.imageson.org/document.html?id=1071).

Godelier Maurice, 1982. La production des Grands Hommes. Pouvoir et domination masculine chez les Baruya de Nouvelle-Guinée, Paris, Fayard.

—, 1992. Corps, parenté, pouvoir(s) chez les Baruya de Nouvelle-Guinée, Journal de la Société des Océanistes 94, pp.3-24 (http:// www.persee.fr/doc/jso_0300-953x_1992_ num_94_1_2603).

—, 2004. Métamorphoses de la parenté, Paris, Fayard.

—, 1967-1980 1988. Archives de terrain non publiées, en accès restreint sur la plateforme ODSAS (http://www.odsas.fr/) Carnets I à Xxxi (1967 à 1980), carnets juillet 1988a, juillet 1988b.

Hage Per et Frank Harary, 1981. Pollution Beliefs in Highland New Guinea, Man (N.s.) 16, pp. 367-375.

Hamberger Klaus, Michael Houseman, Isabelle Daillant, Douglas R. White and Laurent BARRY, 2004. Matrimonial Ring Structures, Mathématiques et Sciences humaines 168, pp. 83-119.

Hamberger Klaus, Michael Houseman et Cyril Grange, 2009. La parenté radiographiée : un nouveau logiciel pour l'analyse des réseaux matrimoniaux, L'Homme 191, pp. 107-138.

Herdt Gilbert, 1981 [reed. de 1994]. Guardians of the Flutes. Idioms of Masculinity, Chicago and London, The University of Chicago Press.

Herdt Gilbert (ed.), 1982. Rituals of Manhood. Male Initiation in Papua New Guinea, Berkeley, University of California Press.

-, 1984. Ritualized Homosexuality in Melanesia, Berkeley, University of California Press.

Héritier Françoise, 1994. Les deux soeurs et leur mère. Anthropologie de l'inceste, Paris, Odile Jacob.

—, 1996 [rééd. de 2002]. Masculin/Féminin I : La pensée de la différence, Paris, Odile Jacob.

-, 2003. Une anthropologie symbolique du corps, Journal des africanistes 73 (2), pp. 9-26. 
Houseman Michael et Douglas R. White, 1996. Structures réticulaires de la pratique matrimoniale, L'Homme 139, pp. 59-85.

Jorgensen Dan, 1983. "The facts of life, Papua New Guinea style”, in Dan Jorgensen (ed.), Concepts of Conception: Procreation Ideologies in Papua New Guinea, pp. 1-12. Mankind special issue 14,1 .

Keesing Roger M., 1982. Introduction, in Gilbert Herdt (ed.), Rituals of Manhood. Male Initiation in Papua New Guinea, Berkeley and Los Angeles, University of California Press, pp. 1-43.

Knauft Bruce M., 1989. Bodily images in Melanesia: cultural substances and natural metaphors, in Michel Feher (ed.), Fragments for a history of the human body: part 3, New York, Zone, pp. 198-279.

-, 1993. South Coast New Guinea Cultures. History, Comparison, Dialectic, Cambridge, Cambridge University Press (Cambridge Studies in Social and Cultural Anthropology 89).

—, 2003. What ever happened to Ritualized Homosexuality? Modern Sexual Subjects in Melanesia and Elsewhere, Annual Review of Sex Research 14, ProQuest Central, pp. 137-159.

Lemonnier Pierre, 1981. Le commerce intertribal des Anga de Nouvelle-Guinée, Journal de la Société des Océanistes 70-71, pp. 39-75 (http://www.persee.fr/doc/jso_0300-953x_ 1981_num_37_70_3048).

_, 1997. "Mipela wan bilas". Identité et variabilité socioculturelle chez les Anga de Papouasie-Nouvelle-Guinée, in Serge Tcherkézoff et Françoise Marsaudon (éds), Le Pacifique-Sud aujourd'hui. Identités et transformations culturelles, Paris, CNRs Éditions, pp. 197-227.

-, 2010. Mythes et rites chez les Anga, Journal de la Société des Océanistes 130-131 : Hommage à Bernard Juillerat, pp. 209-220 (http:// jso.revues.org/6070).

—, 2013. Arcs, flèches et orgue électrique. À propos de modernité et d'offensives évangéliques dans la vallée de Wonenara (Papouasie Nouvelle-Guinée), in Christophe Pons (éd.), Jésus, moi et les autres. La construction collective d'une relation personnelle à Jésus dans les Églises évangéliques: Europe, Océanie, Maghreb, Paris, CNRS Éditions, pp. 189-226.

Malbrancke Anne-Sylvie, 2016. Dix commandements mais pas de Dieu: christianisme et changement socioculturel chez les Baruya (PNG)", Journal de la Société des Océanistes 142-143, pp. 241-256 (https://jso. revues.org/7502).

Mimica Jadran, 1981. Omalyce. An Ethnography of the Iqwaye View of the Cosmos, Ph.D. dissertation, Canberra, Australian $\mathrm{Na}-$ tional University.

Porqueres I Gene Enric, 1998. Lourde Alliance, Mariage et identité chez les descendants de Juifs convertis à Majorque (1435-1750), Paris, Kimé.

Robbins Joel, 2007. Thinking and the Problem of Christian Culture Belief, Time, and the Anthropology of Christianity, Current Anthropology 48 (1), pp. 5-38.

-, 2009. History, cosmology and gender: Christianity and cultural change among the Urapmin, in Pamela J. Stewart et Andrew Strathern (eds), Religious and Ritual Change. Cosmologies and Histories, Durha, Carolina Academic Press, pp. 109-132.

SaHlins Marshall, 1981. Historical Metaphors and Mythical Realities: Structure in the Early History of the Sandwich Islands Kingdom, Ann Arbor, University of Michigan Press.

Strathern Marilyn, 1988. The Gender of the Gift. Problems with Women and Problems with Society, Berkeley, University of California Press.

Tuzin Donald, 1997. The Cassowary's Revenge: The Life and Death of Masculinity in a New Guinea Society, Chicago, University of Chicago Press.

Wagner Roy, 1967. The Curse of the Souw. Principles of Daribi Clan Definition and Alliance in New Guinea, Chicago, University of Chicago Press.

Wardlow Holly, 2006. Wayward Women. Sexuality and Agency in a New Guinea Society, Berkeley, University of California Press. 